\title{
UTILIZAÇÃO DO BIM COMO FERRAMENTA PARA OBTENÇÃO DO SELO CASA AZUL DA CAIXA ECONÔMICA FEDERAL
}

Allan Pereira Gomes da Silva - allannuke@gmail.com

Universidade Federal do Cariri

Juazeiro do Norte - Ce

David Willian Gonçalves Silveira - gdavidwillian@gmail.com

Universidade Federal do Cariri

Juazeiro do Norte - Ce

Fábio Cristian Alves Pinheiro Filho -fabio.cristian@aluno.ufca.edu.br

Universidade Federal do Cariri

Juazeiro do Norte - Ce

Francisco César Silva Coelho Filho -fcocesarcoelho@gmail.com

Universidade Federal do Cariri

Crato - Ce

Igor Antônio Alves Pereira -igorantonio980@gmail.com

Universidade Federal do Cariri

Juazeiro do Norte - Ce

Pedro Henrique Castro do Nascimento - henrikelpn@gmail.com

Universidade Federal do Cariri

Crato - Ce

Ana Verônica Gonçalves Borges - ana.borges@ufca.edu.br

Universidade Federal do Cariri

Juazeiro do Norte - Ce

\section{Resumo}

Nos últimos anos, o mercado da construção civil deparou-se com a necessidade de aliar projetos detalhados e integrados com a realização de empreendimentos mais sustentáveis. Desse modo, a utilização da modelagem da informação da construção (BIM) demonstrou-se uma solução eficaz e inovadora para a realização de projetos, promovendo a integração de todas as etapas de uma obra, bem como apresentando um vasto nível de detalhamento dos componentes. Neste sentido, visando atender as exigências para alcançar o título "construção sustentável", o exposto trabalho demonstra como a metodologia pode auxiliar na obtenção do Selo Casa Azul Caixa. A princípio, apresentam-se as valências da ferramenta, juntamente com os meios de implantá-la em uma empresa. Em seguida, conceitua-se o selo, exibindo a sua definição e apontando os tópicos que compõem as demandas para a sua conquista. Logo após, expõe-se um estudo comparativo entre as categorias que integram a certificação e dispositivos BIM, demonstrando a sua eficácia em concepções de projetos ecológicos. Dessa forma, os parâmetros analisados evidenciam o potencial da metodologia, através de uma implantação eficaz, de atender as demandas do selo ambiental. 
Palavras-chave: BIM. Sustentabilidade. Selos ambientais.

\title{
USE OF BIM AS A TOOL TO OBTAIN THE CASA AZUL SEAL FROM CAIXA ECONÔMICA FEDERAL
}

\begin{abstract}
:
In recent years, the civil construction market has faced the need to combine detailed and integrated projects with the realization of more sustainable buildings. Thus, the use of building information modelling (BIM) proved to be an effective and innovative way for the realization of projects, promoting the integration of all stages of a construction, as well as presenting a vast level of detail of the components. In this sense, in order to meet the requirements to achieve the title "sustainable construction", the exposed work demonstrates how the methodology can help in obtaining the Selo Casa Azul Caixa. At first, the tool's valencies are presented, along with the ways of implementing it in a company. Then, the seal is conceptualized, showing its definition and pointing the topics that compose the demands for the achievements of the stamps. Soon after, a comparative study between the categories that integrate the certification and BIM devices is exposed, demonstrating its effectiveness in conception of ecological projects. Thus, the parameters analyzed show the potential of the methodology, through an effective implementation, to meet the demands of the environmental seal.
\end{abstract}

Keywords: BIM. Sustainability. Environmental seals.

\section{INTRODUÇÃO}

O ramo da construção civil não para de evoluir. A cada ano que passa, novos softwares, novas técnicas e novas filosofias de trabalho surgem, e os profissionais da área precisam evoluir de acordo com as demandas do mercado, essas que mudam de acordo com as necessidades do setor e que vão surgindo dia após dia. Uma das demandas que mais vem surgindo no ramo da engenharia civil é a demanda por construções mais sustentáveis. De acordo com Conto, Oliveira e Ruppenthal (2017, p. 101), "Aproximadamente 35\% do total dos recursos naturais consumidos pelo setor produtivo são associados à construção." Além desse alto consumo de recursos naturais, a construção civil também gera outros impactos ambientais bastante graves, como a geração de resíduos, por exemplo.

Durante muito tempo foi debatido entre os profissionais do ramo, ideias e estratégias para tentar amenizar o impacto que a indústria da construção civil está causando na natureza. Silva, Salgado e Silva (2015, p. 2) afirmam que:

A busca pela produção de edificações que considerem as variáveis de desempenho interno e externo (ou seja, conforto ambiental para os usuários e baixo impacto ambiental na região) tem levado os profissionais da área de gestão e desenvolvimento de projetos à discussão sobre a incorporação desses requisitos não apenas nos novos projetos, mas também nos projetos das edificações existentes.

DOI 10.4322/SINABIM.2020.0021 
Uma atitude que pode contribuir para a incorporação desses requisitos é a adesão das certificações e selos ambientais, que servem para atestar que o empreendimento foi construído usando métodos sustentáveis.

O BIM (Building Information Modeling) é uma filosofia de trabalho que quando utilizada em um empreendimento, acompanha todo o seu desenvolvimento desde o seu planejamento, estando presente em todas as etapas construtivas da edificação. Ele oferece várias ferramentas essenciais na construção da edificação, estando algumas delas diretamente voltadas para o aspecto da sustentabilidade do projeto. Segundo Arnal (2018), o BIM possui no momento um total de 10 dimensões, começando da 1D até a 10D. A dimensão 6D do BIM apresenta ferramentas e conceitos que viabilizam empreendimentos mais sustentáveis, assim facilitando alcançar os pré-requisitos necessários para a obtenção das certificações ambientais.

\subsection{OBJETIVOS}

Este artigo possui como finalidade central abordar a introdução da metodologia BIM em empresas, por meio de um Plano de Implementação BIM (BIP), e a sua contribuição para a obtenção do selo Casa Azul Caixa. No cenário atual da construção civil, nota-se a crescente necessidade de possuir controle sobre todas as etapas da construção e, com isso, atingir um fluxo de trabalho cooperativo e com mais riqueza de detalhes. Logo, a utilização de ferramentas integradas demonstra-se uma solução viável de exigências para a concepção de edifícios verdes.

Sabendo que a metodologia BIM oferece muitas ferramentas para ter um empreendimento mais sustentável, o presente estudo visa apontar, por meio de ferramentas BIM, soluções sustentáveis que atendam aos pré-requisitos para a obtenção do selo Casa Azul Caixa, com o intuito de executar edificações ecologicamente harmônicas e com um sistema produtivo de projetos.

\section{BIM E BIP}

Segundo o BIM dictionary (2020) o BIM é um conjunto de tecnologias, processos e políticas que permitem aos múltiplos interessados de um empreendimento projetar, construir e operar colaborativamente uma construção no espaço virtual.

As aplicações dos variados níveis do paradigma, conforme Eastman, Teicholz, Sacks e Liston (2014) destacaram, são divididas e designadas por dimensões, que estão detalhadas abaixo:

- BIM 2D: Relacionado a parte de documentação e dos planos de ação, tais como BEP (Plano de Execução BIM) e BIP (Plano de Implementação BIM).

- BIM 3D: Consiste na modelagem dos elementos que constituem a edificação e diferentemente das representações gráficas já utilizadas, no BIM 3D, cada elemento tem informações consideráveis para os processos construtivos seguintes.

- BIM 4D: O modelo 4D conforme Guimarães (2017) é dinâmico e por meio de uma variação do tempo, permite que o modelo 3D seja variável de acordo com as exigências do observador, proporcionando a visualização da construção em qualquer momento de acordo com seu cronograma.

DOI 10.4322/SINABIM.2020.0022 
- BIM 5D: Inclui na modelagem do projeto, a orçamentação e quando integrado ao gerenciamento 4D da obra, possibilita visualizar a execução de cada atividade e o custo envolvido com o tempo;

- BIM 6D: Integra na modelagem 3D em união com as outras dimensões, tecnologias a favor da sustentabilidade tais como análises energéticas através de georreferenciamento e características climáticas.

Apesar de o BIM possuir muitas possibilidades de melhorias nos projetos, sua adoção pelas empresas no Brasil permanece aquém do esperado, se comparado aos países que já o adotam. Diante dessa realidade, a Câmara Brasileira da Indústria da Construção (CBIC), por meio do segundo volume da Coletânea Implementação do BIM para Construtoras e Incorporadoras, aponta 4 motivos que explicam porque essa adoção no Brasil não se dá de forma mais rápida:

- Inércia e resistência às mudanças: Para que uma mudança aconteça de fato numa empresa ou organização, são necessários cinco componentes críticos: visão, capacitação, incentivos, recursos e o desenvolvimento de um plano de ação, caso algum desses esteja em falta, há o risco de uma implantação mal sucedida.

- Dificuldade de entendimento e compreensão: Uma impressão equivocada, mas bastante disseminada, é que o BIM seria um 'substituto do CAD', quando é, na verdade, uma inovação tecnológica muito mais profunda, pois altera radicalmente todo o processo de projeto, desde a concepção até o gerenciamento do empreendimento.

- Barreiras culturais e particularidades do ambiente brasileiro: $\mathrm{O}$ atual modelo de contratação de projetistas utilizado no Brasil. Os maiores beneficiados pela adoção BIM são os contratantes, que respondem pelo produto final construído perante os clientes. Para os projetistas, entretanto, a exigência do BIM acaba representando, além da necessidade de investimento e capacitação, um aumento substancial de escopo e responsabilidades, sem que sua remuneração seja adequadamente revista.

- Especificidades e aspectos intrínsecos ao BIM: A adoção BIM requer esforço, aprendizado e investimento. Não é simples comprovar o retorno sobre o investimento (ROI) no BIM. É difícil mensurar alguns dos principais benefícios oferecidos pelo BIM, que é justamente o aumento da precisão dos projetos e do planejamento.

Visto isso, para uma empresa que de fato entende que o BIM vai além da aquisição de um determinado software e que está ciente da mudança na cadeia de processos, o BIP (Plano de Implementação BIM) entra como ferramenta de implementação do paradigma de forma mais eficaz no âmbito empresarial.

O BIP, segundo CBIC (2016), é feito através de um profissional especialista na área, de preferência um BIM Manager (Gerente BIM), pois é um processo complexo e detalhado, cuja elaboração varia de acordo com a realidade e demanda de cada empresa, entretanto, via de regra ele segue alguns passos que são comuns na definição do escopo do plano a ser adotado.

\section{SELO CASA AZUL}


O Selo Casa Azul é uma medida nacional de avaliação de desempenho ambiental de edificações habitacionais, aplicável a todos os tipos de projetos nas linhas de financiamento da Caixa que almejam a obtenção do selo. De acordo com o Guia Selo Casa Azul Caixa (2020), foi criado em 2009 sendo o primeiro sistema de classificação com desenvolvimento pleno brasileiro levando em conta suas particularidades, como por exemplo, os fatores sociais que são fundamentais no processo sustentável.

O sistema de classificação foi totalmente reformulado em 2019. Atualmente sua versão, de junho de 2020 possui 4 níveis de classificação: bronze, prata, ouro e diamante, que são atingidos de acordo com sua pontuação nos critérios de avaliação que estão representados abaixo:

Tabela 1 - Categorias do Selo Casa Azul

\begin{tabular}{|c|c|}
\hline ITEM & CATEGORIA \\
\hline 1 & Qualidade urbana e bem estar \\
\hline 2 & Eficiência energética e conforto ambiental \\
\hline 3 & Gestão eficiente da água \\
\hline 4 & Produção sustentável \\
\hline 5 & Desenvolvimento social \\
\hline 6 & Inovação \\
\hline
\end{tabular}

Fonte - Adaptado de Guia Selo Casa Azul, 2020

A CAIXA tem função de fornecer e orientar o preenchimento de todas as documentações necessárias, bem como de fiscalizar o projeto e vistoriar sua execução. Após aprovação o certificado é emitido de acordo com seu nível de classificação.

Um dos benefícios da certificação é seguir as tendências de mercado onde mais consumidores buscam e exigem projetos mais sustentáveis, uma vez que, além de mais respeito ao meio ambiente, também garantem uma qualidade de vida mais satisfatória. É interessante frisar também, que este tipo de certificação pode garantir um diferencial importante em relação à concorrência.

Existem também incentivos fiscais para o construtor onde serão avaliados descontos nas taxas de juros de financiamento, bem como benefício para o comprador com a possibilidade de taxas de juros diferenciadas para adquirir unidades de empreendimentos com a certificação.

Mesmo que alguns critérios exijam um custo inicial mais alto, isso resultará em vantagens posteriormente. Verificar impactos sobre a qualidade do entorno e avaliar a eficiência energética são, por exemplo, alguns dos critérios obrigatórios, assegurando uma maior longevidade a obra e um consumo racional de água e energia.

Dessa forma, podemos destacar que a obtenção do Selo Casa Azul busca incentivar a prática sustentável na construção de novas edificações, resultando assim em mais qualidade 
para o resultado final da obra, o que também garante mais valorização, satisfação e economia para o empreendimento e o adquirente.

\section{METODOLOGIA}

A metodologia utilizada foi a pesquisa bibliográfica que, segundo Boccato (2006, p. 266), busca a resolução de problemas em referenciais teóricos publicados, analisando e discutindo as várias contribuições científicas, trazendo subsídios para o conhecimento sobre o que foi pesquisado, como e sob que enfoque e/ou perspectivas foi tratado o assunto apresentado na literatura científica.

A metodologia consistiu na análise do selo Casa Azul Caixa, junto aos seus critérios de obtenção, atrelado ao conceito BIM. Criado em 2009 e reformulado em 2019, esse selo requer que uma série de pré-requisitos seja atendida. Dessa forma, através do guia disponibilizado pela Caixa e conceitos BIM, foi analisado como unir a sustentabilidade, ferramentas BIM, e os critérios do selo, buscando facilitar a elaboração e execução de obras mais eficientes.

\section{RESULTADOS E DISCUSSÕES}

A partir da comparação entre a metodologia BIM e os critérios necessários para obtenção do Selo Casa Azul percebe-se que caso ocorra a implementação do BIP nas empresas, os empreendimentos construtivos terão um grande ganho de produtividade e de acerto quanto aos requisitos para obtenção do selo casa azul. A partir do que foi analisado, segue abaixo um paralelo entre alguns dos critérios para a edificação obter o selo casa azul e o que a implementação do BIM pode agregar.

- Qualidade Urbana e Bem estar: Concluindo o BIP e fazendo o uso da dimensão 3D do BIM, é possível realizar uma melhor análise topográfica do terreno, verificar as questões de infraestrutura (pavimentação, saneamento e iluminação pública). Além da documentação dos parâmetros adotados e das referências de forma eficiente.

- Eficiência energética e conforto ambiental: Com a implementação do BIM e fazendo o uso da sua sexta dimensão, será possível a realização de simulações ambientais dentro de softwares garantindo um maior conforto ambiental, pois a partir dessas simulações pode-se ter uma verificação da irradiação solar e das massas de vento na edificação. Ademais, é possível realizar um maior controle dos materiais utilizados na construção, visando utilizar materiais que tenham uma melhor eficiência energética como também a verificação de equipamentos que tenham um menor consumo energético e, assim, diminuindo a demanda ao sistema de distribuição de energia elétrica. Como alternativa futura para as edificações, o BIM também fornece a possibilidade da realização de um controle de processos e manutenção podendo futuramente, com a obsolescência de alguns equipamentos instalados, realizar o que é chamado de "retrofit", que significa a troca por equipamentos mais modernos e mais eficientes energeticamente.

- Gestão eficiente da água: A partir das dimensões 3D e 6D será possível a criação de famílias paramétricas que tenham os dados relacionados ao consumo de água de cada

DOI 10.4322/SINABIM.2020.0025 
equipamento. Dessa forma, sendo possível analisar qual modelo e quais equipamentos têm um menor consumo de água.

- Produção sustentável: O BIM tem como propósito, na quarta e quinta dimensão, o controle de custos, materiais e etapas da obra, o que pode diretamente influenciar em uma produção com uma redução de resíduos sólidos advindos da construção e assim obtendo uma produção sustentável da construção.

- Desenvolvimento social: Ainda em estudo dentro da metodologia BIM, existe a possibilidade da implantação da Segurança e Saúde no canteiro de obras realizado a partir de um controle automatizado e virtual, possibilitando um maior controle das áreas de risco, dos equipamentos de proteção individual e coletivos como também das medidas de controle a serem tomadas dentro do canteiro em casos de acidentes.

- Inovação: Além dos vários critérios citados nos tópicos anteriores quanto ao BIP, a própria implementação do BIM na gestão integrada do empreendimento se encaixa como inovação para a aquisição do Selo Caixa Azul,

\section{CONCLUSÕES}

A implementação do BIM a partir do BIP é de grande importância para a obtenção de dados seguros e de qualidade. Para isso, torna-se indiscutível a figura dos gerentes BIM dentro das empresas, sendo estes os responsáveis por adequar a implementação do BIM a cada realidade encontrada. Percebe-se também a possibilidade da própria Caixa Econômica Federal implementar o BIM, já que esta é responsável por fiscalizar e vistoriar a execução dessas obras.

Além disso, foi demonstrado que com a tecnologia atual dos softwares e metodologias BIM que fazem parte do mercado brasileiro, é possível uma empresa implementar e atender a grande maioria das exigências do Selo Casa Azul, além de facilitar e agilizar as análises, visto que os modelos em BIM contém maior riqueza de informações e detalhes.

Entretanto, a implementação do BIM de forma plena no mercado brasileiro também depende de mudanças culturais e práticas de contratação que divergem dos modelos atuais, bem como o amadurecimento de conceitos BIM na comunidade da construção civil de modo a evitar expectativas exageradas e também um maior foco de utilização da metodologia de acordo com a realidade de cada empresa/profissional.

\section{AGRADECIMENTOS}

Aos membros da Liga Acadêmica de Building Information Modeling (LABIM) da UFCA por prestar apoio técnico e humano na concepção e desenvolvimento desta pesquisa.

\section{REFERÊNCIAS}


ARNAL, Ignasi Pérez. Why don't we start at the beginning? The Basics of a Project: Lean Planning and Pre-Construction, BIM News Last trends of the AECO sector, BIM Community, 2018.

BOCCATO, V. R. C. Metodologia da pesquisa bibliográfica na área odontológica e o artigo científico como forma de comunicação. Rev. Odontol. Univ. Cidade São Paulo, São Paulo, v. 18, n. 3, p. 265-274, 2006.

BUILDING Information Modeling. BIM dictionary. Disponível em https://bimdictionary.com. Acesso em: 04 ago. 2020

CAIXA ECONÔMICA FEDERAL. Guia Selo Casa Azul (2020). Disponível em: http://www.caixa.gov.br/Downloads/selo_casa_azul/Guia_Selo_Casa_Azul_CAIXA_Junho_2 020.pdf. Acesso em: 01 ago. 2020.

CÂMARA BRASILEIRA DA INDUSTRIA DA CONSTRUÇÃO. Implementação do BIM para Construtoras e Incorporadoras Vol.2, 2016.

CONTO, V.; OLIVEIRA, M. L.; RUPPENTHAL, J. E. Certificações ambientais: contribuição à sustentabilidade na construção civil no Brasil. GEPROS. Gestão da Produção, Operações e Sistemas, Bauru, Ano 12, nº 4, out-dez/2017, p. 100-127.

EASTMAN, C.; TEICHOLZ, P.; SACKS, R.; LISTON, K. Manual de BIM: Um guia de modelagem da informação da construção. Tradução: Cervantes Gonçalves Ayres Filho et al. Porto Alegre: Bookman, p. 84-86, 2014.

GUIMARÃES, Amanda Matsui. Aplicação do BIM 4D para a Otimização do Cronograma Físico de uma Obra. 2017. 69f. Monografia (Graduação em Engenharia Civil) Universidade Federal de Goiás. Goiânia, 2017.

SILVA, F.; SALGADO, M.; SILVA, C. Plataforma BIM, Retrofit e Sustentabilidade Ambiental: Estudo de Caso no Rio de Janeiro. In: ENCONTRO BRASILEIRO DE TECNOLOGIA DE INFORMAÇÃO E COMUNICAÇÃO NA CONSTRUÇÃO, 7., 2015, Recife. Anais... Porto Alegre: ANTAC, 2015. p. 1-12. 